\title{
CARINA:
}

\section{a European network for nuclear astrophysics}

\author{
Carmen Angulo (for the CARINA network)* \\ Institut de Physique Nucléaire et Centre de Recherche du Cyclotron, \\ Université catholique de Louvain, B-1348 Louvain-la-Neuve, Belgium \\ E-mail: angulo@cyc.ucl.ac.be
}

\begin{abstract}
Nuclear astrophysics has revealed as one of the most exciting areas of interdisciplinary research, in the crossroads of nuclear physics, astrophysics and astronomy. As a driving force for the development of the very first Radioactive Ion Beam (RIB) Facilities, it remains a fundamental research area in many European laboratories and is one of the key research topics in future RIB Facilities in Europe. Coupled to these innovative experimental efforts are substantial communities of theorists, astrophysicists and observational astronomers. Input from all of these sectors is necessary to achieve realistic modeling of stellar environments, the ultimate aim being a thorough understanding of the abundance and evolution of the elements and of the processes of energy generation in the Universe.

Within the EURONS project (2005-2008), the CARINA network intends to provide coherence to the research activities in nuclear astrophysics in Europe. The main objetives and activities of this network are presented here. Updated information is available at the website: www.cyc.ucl.ac.be/CARINA.
\end{abstract}

International Symposium on Nuclear Astrophysics - Nuclei in the Cosmos - IX

June 25-30 2006

CERN, Geneva, Switzerland

*This work is supported by the European Commission within the Sixth Framework Programme through I3EURONS (contract no. RII3-CT-2004-506065). 


\section{Motivation}

Nuclear astrophysics involved careful and dedicated experimental and theoretical studies of a large variety of nuclear processes (nuclear physics) as indispensable tools for the modeling of stellar evolution and nucleosynthesis (astrophysics). Although the human potential in nuclear astrophysics research in Europe is considerable, the effort is still dispersed. The European impact in this research field could be greatly enhanced by a concerted interdisciplinary effort directly bringing together experts in nuclear physics theory, stellar modelling and experimental techniques.

Within the EURONS initiative ${ }^{1}$, the CARINA network intends to provide coherence to the research activities in nuclear astrophysics in Europe. CARINA stands for Challenges and Advanced Research In Nuclear Astrophysics. The central objective of this network is to identify the key forefront studies in this research area and to provide the necessary guidance to European laboratories, assuring the best development and usage of experimental facilities.

\section{Objetives and main activities}

The main objetives of the CARINA network can be summarised as follows:

- To identify the available instrumentation and human potential in Europe by carrying out mapping studies in terms of projects, facilities and teams.

- To develop the research capabilities of the nuclear physics Large Scale Facilities and of the other laboratories and research groupes working in nuclear astrophysics.

- To enhance involvement in the future radioactive and stable beam facilities.

- To coordinate research efforts by defining and proposing common research goals and by encouraging new collaborations and $\mathrm{R} \& \mathrm{D}$ projects.

- To record the needs for new instrumentation and novel techniques by looking for existing solutions in other fields.

In order to facilitate these complex activities, four working groups (WG), namely WG1: Astrophysics and Nuclear Theory, WG2: Instrumentation, WG3: Link to the EURONS LSF, and WG4: Experimental groups have been established. Each WG is coordinated by two members of the nuclear(astro) physics community. The conveners of the WG's have the explicit role of: (i) informing the CARINA members of the different activities in their specific area in Europe,(ii) serving as a link between CARINA and the EURONS community, (iii) coordinating initiatives related to their area within CARINA, and (iv) assisting the CARINA coordinator (reports, proposals, common initiatives, webpage...).

\footnotetext{
${ }^{1}$ EURONS, EUROpean Nuclear Structure, is the first common project at the European level (more than 50 institutions from 21 European countries) supporting nuclear physics research around eight Large Scale Facilities. More information can be found in the website: www.gsi.de/eurons.
} 
At present, scientists from 31 research groups from $13 \mathrm{EU}$ and Associated countries are involved in CARINA. They are nuclear physicists (experimentalists and theoreticians), astrophysicists and observational astronomers, as well as representatives of the EURONS Large Scale Facilities: CRC-UCL (Belgium), CERN-ISOLDE (Europe), GSI (Germany), GANIL (France), JYUJYFL (Finland), INFN-LNL (Italy) and, RUG-KVI (Netherlands). Information on the network participants can be found in the CARINA website: www.cyc.ucl.ac.be/CARINA, where updated information concerning the network activities (working groups, documents, presentations, workshops, collaborations, etc) is also available.

In particular, a new collaborative effort between CARINA and EURISOL has been recently established. The CARINA coordinator is also the leader of the subtask Astrophysics in the EURISOL Task 10 Physics and Instrumentation. More information can be found in the website: http://ns.ph.liv.ac.uk/eurisol or through the CARINA website. In addition, the CARINA network supports the efforts for the construction of a dedicated high intensity stable heavy ion beam facility and a complementary high-current low-energy accelerator laboratory in Europe thought the ECOS collaboration.

The members of the nuclear astrophysics community interested on the CARINA activities should contact the convevers of their specific area or, directly, the CARINA coordinator.

\section{Perspectives}

The final goal of the CARINA network is to strengthen the research capabilities of nuclear astrophysics in the programs of the present European laboratories and of the future second-generation facilities. With this aim, and having in mind the next EC framework program FP7, CARINA intends to promote the establishment of new joint research projects, networks and/or other activities that can enhance in a considerable manner the human potential and the long-term plans of nuclear astrophysics research in Europe. 